\title{
From Brain Signals to Adaptive Interfaces: using fNIRS in HCI
}

\author{
Audrey Girouard*, Erin Treacy Solovey*, Leanne M. Hirshfield*, Evan M \\ Peck*, Krysta Chauncey*, Angelo Sassaroli+, Sergio Fantini+ and Robert J. \\ K. Jacob* \\ *Computer Science Department +Biomedical Engineering Department \\ Tufts University \\ Medford, MA 02155, USA \\ \{audrey.girouard, erin.solovey, leanne.hirshfield, evan.peck, krysta.chauncey, \\ angelo.sassaroli, sergio.fantini, robert.jacob\} @ tufts.edu
}

\begin{abstract}
Functional near-infrared spectroscopy (fNIRS) is an emerging noninvasive, lightweight imaging tool which can measure blood oxygenation levels in the brain. In this chapter, we describe the fNIRS device and its potential within the realm of human-computer interaction (HCI). We discuss research that explores the kinds of states that can be measured with fNIRS, and we describe initial research and prototypes that can use this objective, real time information about users' states as input to adaptive user interfaces.
\end{abstract}

\section{Introduction}

The field of brain-computer interfaces (BCI) is beginning to expand beyond its original clinical focus. While traditional BCIs were designed to function using the brain as the sole means of communication for disabled patients (Millán, Renkens, Mouriño, \& Gerstner, 2004), current research investigates the brain as an additional input to the interface, designed for a broader range of users (Grimes, Tan, Hudson, Shenoy, \& Rao, 2008). Neural signals can act as a complementary source of information when combined with conventional computer inputs such as the mouse or the keyboard. We present work in this chapter that illustrates this direction in BCI and shows how to move from controlled experiments exploring taskspecific brain activity to a more general framework using mental activity to guide interface response. Our work, grounded in the field of human-computer interaction (HCI), suggests the practicality and feasibility of using normal untrained brain activity to inform interfaces.

While most BCIs use the electroencephalogram (EEG) to measure brain activity, we adopt the relatively less-explored technique of functional near-infrared spectroscopy (fNIRS), a non-invasive measurement of changes in blood oxygena- 
tion, which can be used to extrapolate levels of brain activation (Fig. 1). Ideally, for HCI research, the fNIRS signals would be robust enough to remain unaffected by other non-mental activities, such as typing, occurring during the participant's task performance. In fact, one of the main benefits of fNIRS is that the equipment imposes few physical or behavioral restrictions on the participant (Hoshi, 2009).

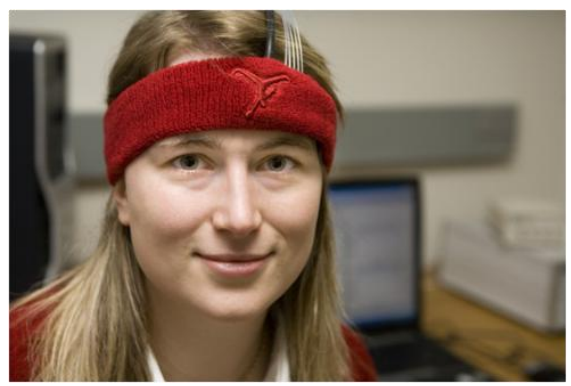

(a)

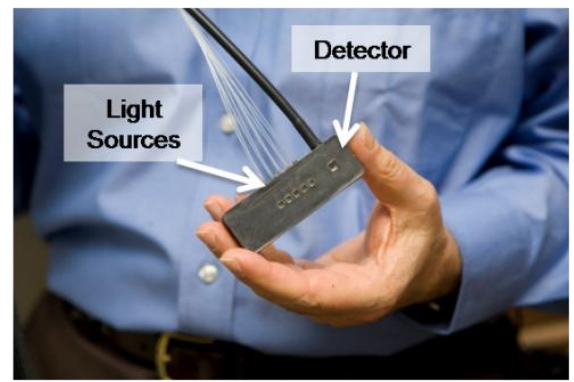

(b)

Fig. 1. Functional near-infrared spectroscopy: (a) typical setup with a sensor secured on forehead with a headband, (b) the sensor containing near-infrared light sources and a light detector.

We identify two research questions that shape this chapter: (1) what kind of states can we measure using fNIRS? (2) how should we use this information as input to an adaptive user interface?

To address our first question, we will start by discussing practicality and applicability of the technology in realistic, desktop environments (Solovey, et al., 2009). We will then describe studies investigating the use of fNIRS to obtain meaningful data related to mental workload, both with workload as an overall cognitive function (Girouard, et al., 2009; Hirshfield, et al., 2007), and with specific components of it (Hirshfield, et al., 2009b). Our studies progress from very controlled experiments that help us identify centers of brain activity, to experiments using simple user interfaces, showing how this technique may be applied to more realistic interfaces. Throughout all studies in this chapter, we show the use of novel machine learning techniques applied to fNIRS, in order to classify and use the brain activity information in real time.

Our second goal focuses on creating new interactive, real-time user interfaces, which can adapt behavior based on brain measurements. The design challenge is to use this information in a subtle and judicious way, as an additional, lightweight input that could make a mouse or keyboard-driven interface more intuitive or efficient. Specifically, we are exploring situations and interfaces that can be adapted slowly, in a manner that is subtle and unobtrusive to the user, which could increase productivity and decrease frustration. At the end of this chapter, we describe two early prototypes of user interfaces that can adapt to the user's workload profile or other brain activity in real time. 


\section{2 fNIRS Background}

fNIRS provides a measure of blood oxygen concentration, indicative of brain activity when measured on the head (Villringer \& Chance, 1997). Near-infrared light is pulsed into the forehead where it is refracted from the tissues of the cortex up to depths of $1-3 \mathrm{~cm}$. Oxygenated and deoxygenated hemoglobin are the main absorbers of light at these wavelengths, and thus the diffusely reflected light picked up by the detector correlates with the concentration of oxygen in the blood, as well as the overall amount of blood in the tissue. The basic technology is common to all systems, but the measured signal differs depending on the location of the probe and the amount of light received.

There are many possible placements of fNIRS probes, allowing the study of multiple brain regions. The most common placements are on the motor cortex (Sitaram, et al.), and the prefrontal cortex (PFC) (Ehlis, Bähne, Jacob, Herrmann, \& Fallgatter, 2008; Mappus, Venkatesh, Shastry, Israeli, \& Jackson, 2009), although other regions have also been explored (Herrmann, et al., 2008). We chose to study the anterior PFC ( $\mathrm{aPFC}$ ), an active region that deals with high-level processing such as working memory, planning, problem solving, memory retrieval and attention (Ramnani \& Owen, 2004). Thus, our considerations below are intended for researchers investigating the aPFC; methods that reflect activity in other parts of the brain will vary considerably. However, we expect our results to be valid for other experimental setups and contexts that use the PFC.

\section{3 fNIRS Considerations for HCI Research}

Because most brain imaging and sensing devices were developed for clinical settings, they often have characteristics that make them less suitable for use in realistic HCI settings. For example, although functional magnetic resonance imaging (fMRI) is effective at localizing brain activity, it is susceptible to motion artifacts, and even slight movement (more than $3 \mathrm{~mm}$ ) will corrupt the image. The most common technology used for brain measurement in HCI is EEG, since it is noninvasive, portable, and relatively inexpensive compared with other brain imaging devices (Lee \& Tan, 2006). EEG is not ideal for HCI, either: it is susceptible to artifacts from eye and facial movement, as well as nearby electronic devices, requires gel in the participant's hair, and takes time to set up properly.

Recently, fNIRS has been used in HCI because it has many characteristics that make it suitable for use outside of clinical settings (Girouard, et al., 2009; Hirshfield, et al., 2009b; Mappus, et al., 2009). Benefits include ease of use, short setup time, and portability, making it a promising tool for HCI researchers. In addition, there are no technical restrictions for using EEG and fNIRS together (Hirshfield, et al., 2009a), and the two technologies could complement one anoth- 
er. While EEG measures electricity - a direct result of neuronal activity - and is thus very fast, it is also spatially indeterminate; as previously mentioned, fNIRS measures blood oxygenation, an indirect measure of neuronal activity which is much slower to change than the EEG signal.

Our goal is to observe brain signals that can be used in a relatively ordinary computer task environment; so we do not expect the participant to be physically constrained while using the computer. In most studies using brain sensors, researchers expend great effort to reduce the noise picked up by the sensors. Typically, participants are asked to remain still, avoid head and facial movement, and use restricted movement when interacting with the computer. In addition, many factors cannot be controlled, so researchers sometimes throw out data that may have been contaminated by environmental or behavioral noise, or they develop complex algorithms for removing the noise from the data. By doing this, the researchers hope to achieve higher quality brain sensor data, and therefore better estimates of cognitive state information. However, it is not clear that all of these factors contribute to problems in fNIRS data or that these restrictions improve the signal quality, so a series of experiments were conducted in order to observe directly the effect of such artifacts on the data. Below, we identify several fNIRS considerations and explain best practices for working with fNIRS in HCI research.

\section{Head Movement}

Several fNIRS researchers have brought attention to motion artifacts in fNIRS sensor data, particularly those from head movement (Devaraj, Izzetoglu, Izzetoglu, \& Onaral, 2004; Matthews, Pearlmutter, Ward, Soraghan, \& Markham, 2008). They note that these issues are significant if the head is not restricted, and even more so in an entirely mobile situation. However, other researchers indicate that fNIRS systems can "monitor brain activity of freely moving subjects outside of laboratories" (Hoshi, 2009). While fNIRS data may be affected by head movements, they only become a problem when present at a fairly gross level; this should be contrasted with fMRI where movement over $3 \mathrm{~mm}$ will blur the image. Because of the lack of consensus in the community, we investigated the artifacts associated with head movements during typical computer usage to determine their effect on fNIRS sensor data in a typical HCI setting (Solovey, et al., 2009). From our experiments, we suggest that participants minimize major head movements, although we believe these artifacts may be corrected using filtering techniques.

\section{Facial Movement}

fNIRS sensors are often placed on the forehead, and as a result, it is possible that facial movements could interfere with accurate measurements. Coyle, Ward, and Markham (2004) point out that "slight movements of the optodes on the scalp can 
cause large changes in the optical signal, due to variations in optical path". These forehead movements could be caused by talking, smiling, frowning, or by emotional states such as surprise or anger, and many researchers have participants refrain from moving their face, including talking (Chenier \& Sawan, 2007). However, as there is little empirical evidence of this phenomenon, we examined it further (Solovey, et al., 2009).

We found that frowning data could always be distinguished from nonfrowning. We also learned that if all the data includes frowns, then we cannot differentiate the cognitive task from the rest condition. However, we found that if we combine the data that contains frowning and that without frowning, we can then discriminate the cognitive task, which shows interesting potential to identify which examples to reject because of frowns. Those results clearly indicate that frowning is a problematic artifact, and should be avoided as much as possible.

Because fNIRS sensors are placed on the forehead, frowning was expected to have a large impact on this data. It is possible that other facial movements would not have as strong of an effect. Eye movements and blinking are known to produce large artifacts in EEG data, which leads to the rejection of trials; experimenters often ask their participants to refrain from blinking entirely, or to blink during a specific, non-critical period in each trial (Izzetoglu, Bunce, Onaral, Pourrezaei, \& Chance, 2004). In contrast to EEG, fNIRS is less sensitive to muscle tension and researchers have reported that no artifact is produced in nearby areas of the brain (Izzetoglu, et al., 2004). It would also be unrealistic to prevent eye blinks and movement in HCI settings. Overall, we conclude eye artifacts and blinks should not be problematic for fNIRS, and we do not constrain participants in our studies.

\section{Ambient Light}

Because fNIRS is an optical technique, light in the environment could contribute to noise in the data. Coyle, Ward, and Markham (2004) advise that stray light should be prevented from reaching the detector. Chenier and Sawan (2007) note that they use a black hat to cover the sensors, permitting the detector to only receive light from the fNIRS light sources.

While this is a concern for researchers currently using raw fNIRS sensors that are still under development, we feel that future fNIRS sensors will be embedded in a helmet or hat that properly isolates them from this source of noise. Therefore, we have not examined how the introduction of light can affect fNIRS data. Instead we caution that excess light should be kept to a minimum when using fNIRS, or the sensors should be properly covered to filter out the excess light. 


\begin{abstract}
Ambient Noise
During experiments and regular computer usage, one is subjected to different sounds in the environment. Many studies using brain sensors are conducted in sound-proof rooms to prevent these sounds from affecting the sensor data (Morioka, Yamada, \& Komori, 2008). However, this is not a realistic setting for most HCI research. Therefore, we conduct all of our studies in a setting similar to a normal office. It is mostly quiet (although the room is not soundproof), and there may be occasional noise in the hallway, or from climate control systems. We have successfully run many studies using the fNIRS system in these conditions.
\end{abstract}

\title{
Respiration and Heartbeat
}

The fNIRS signals picks up respiration and heartbeat, by definition, as it measures blood flow and oxygenation (Coyle, et al., 2004; Matthews, et al., 2008). These systemic noise sources can be removed using validated filtering techniques. For a discussion of techniques, see Matthew et al. (2008) and Coyle et al. (2004).

\section{Muscle movement}

In clinical settings, it is reasonable to have participants perform purely cognitive tasks while collecting brain sensor data. This allows researchers to learn about brain function without interference from other factors such as muscle movement. However, to move brain imaging methods into HCI settings, this constraint would have to be relaxed, or methods for correcting the artifacts must be developed.

One of the main benefits of fNIRS is that the setup does not physically constrain participants, allowing them to use external devices such as a keyboard or mouse. In addition, motion artifacts are expected to have less of an effect on the resulting brain sensor data (Girouard, et al., 2009). We examined physical motions that are common in HCI settings, typing and mouse clicking, to determine whether they are problematic when using fNIRS (Solovey, et al., 2009).

Overall, while typing artifacts could be detected when there was a cognitive task being performed, we could still distinguish the cognitive task itself from a rest state. This confirms our hypothesis and validates that typing is an acceptable interaction when using fNIRS. From this, we can also assume that simple key presses (e.g. using arrow keys) would also be acceptable with fNIRS since it is a more limited movement than typing with both hands.

We found that mouse clicking might affect the fNIRS signal we are collecting. When the participant was at rest, we found a significant difference in the signal between the presence and absence of clicking. The difference in activation is not surprising as we did not have a "random clicking" task, but one where subject had to reach targets, which may have activated the area being probed (the anterior pre- 
frontal cortex). However, because we still were able to distinguish the cognitive task from rest, the cognitive task may produce a different signal from clicking. Hence, results indicate that when we want to observe a cognitive task that contains clicking, we need to have the rest task contain clicking as well. Overall, we believe that mouse clicking is acceptable if the experiment is controlled.

\section{Slow Hemodynamic Response}

The slow hemodynamic changes measured by fNIRS occur in a time span of 6-8 seconds (Bunce, Izzetoglu, Izzetoglu, Onaral, \& Pourrezaei, 2006). This is important when designing interfaces based on fNIRS sensor data, as the interface would have to respond in this time scale. While the possibility of using eventrelated fNIRS has been explored (Herrmann, et al., 2008), most studies take advantage of the slow response to measure short term cognitive state, instead of instantaneous ones.

\section{Summary of Guidelines and considerations}

According to our research, mouse clicking and typing are not problematic, but large-scale head and facial movements should be minimized; minor movements as well as heartbeat and respiration can be corrected using filtering techniques. Many limitations that are inherent to other brain sensing and imaging devices such as long setup time, highly restricted position, and intolerance to movement are not factors when using fNIRS. By using the guidelines described above, researchers can detect aspects of the user's cognitive state in realistic HCI laboratory conditions. In our work, we have focused on mental workload, and have conducted several experiments described below exploring the feasibility of recognizing mental workload states with fNIRS.

\section{Measuring Mental Workload}

Acquiring measurements about the mental state of a computer user is valuable in $\mathrm{HCI}$, both for evaluation of interfaces and for real time input to computer systems. Although we can accurately measure task completion time and accuracy, measuring factors such as mental workload, frustration and distraction are typically limited to qualitatively observing users or administering subjective surveys. These surveys are often taken after the completion of a task, potentially missing valuable insight into the user's changing experiences throughout the task. They also fail to capture internal details of the operator's mental state. 
As HCI continues to move out of the workplace and into the real world, users' goals and uses for computers are changing and the analysis involved in the evaluation and design of new interfaces is shifting from "usability analysis to user experience analysis" (Mandryk, Atkins, \& Inkpen, 2006). New evaluation techniques that monitor user experiences while working with computers are increasingly necessary. To address these evaluation issues, current research focuses on developing objective techniques to measure in real time user states such as workload, emotion, and fatigue (Gevins \& Smith, 2003; John, Kobus, Morrison, \& Schmorrow, 2004; Marshall, Pleydell-Pearce, \& Dickson, 2003). Although this ongoing research has advanced user experience measurements in the HCI field, finding accurate, non-invasive tools to measure computer users' states in real working conditions remains a challenge. Measuring user workload with objective measures such as galvanic skin response (John, et al., 2004), EEG (Gevins \& Smith, 2003), ERP (Kok, 1997), pupil dilation (Iqbal, Zheng, \& Bailey, 2004), and facial EMG (Fuller, Sullivan, Essif, Personius, \& Fregosi, 1995) has been a topic of much research. It is well understood that a reliable measure of user workload could have a positive impact in many real life interactions (Guhe, et al., 2005; Iqbal, et al., 2004; John, et al., 2004).

We conducted a study to demonstrate the feasibility and potential for using fNIRS in HCI settings (Hirshfield, et al., 2007). We distinguished several discrete levels of workload that users experienced while completing different tasks. In this experiment, four subjects completed thirty tasks where they viewed all sides of a rotating three-dimensional (3D) shape comprised of eight small cubes. The cubes could be colored with two, three, or four colors, which we hypothesized would lead to different workload levels. During each task, subjects counted the number of squares of each color displayed on the rotating shape in front of them. A blank screen represented the baseline state (no colors).

The main goal of this experiment was to establish whether fNIRS data is sufficient for determining the workload level of users as they perform tasks. To accomplish this, a graphical interface displayed the rotating shapes.

At the completion of each task, the subject was prompted for his or her count for each color. Then, the subject rested for thirty seconds, allowing the brain to return to a baseline state. After completing the tasks, the subject was presented with an additional example of each workload level and asked to fill out a NASA-Task Load Index (Hart \& Staveland, 1988), administered to compare our results with an established measure of workload. The results of the NASA-TLX assessment validate our manipulation of workload levels: increased number of colors led to higher workload level.

We classified the data with a multilayer perceptron classifier using the sliding windows approach (Dietterich, 2002). We tested distinguishing all four workload levels from each other, as well as comparisons of two, three, and four workload conditions of the graphical workload level. When we consider the results comparing workload levels 0,2 , and 4, classification accuracies range from $41.15 \%$ to $69.7 \%$ depending on the subject. Considering that a random classifier would have 
$33.3 \%$ accuracy, the results were promising. We could predict, with relatively high confidence, whether the subject was experiencing no workload (level zero), low workload (level two), or high workload (level four).

Our goal was to test the ability of the fNIRS device to detect levels of workload in HCI, to develop classification techniques to interpret its data, and to demonstrate the use of fNIRS in HCI. Our experiment showed several workload comparisons with promising levels of classification accuracy.

\section{Separating Semantic and Syntactic Workload in the Brain}

In our initial work described above, we verified that fNIRS sensors provide researchers with a measure of the mental workload experienced by users working on a task with a given user interface (UI). However, detecting a high workload while a user works with a system is not necessarily a bad thing; it could indicate that the user is immersed in the task. How can UI evaluators and designers of adaptive systems know if a high workload measurement is due to the UI or to the underlying task?

To solve the question, we were influenced by Shneiderman's theory of semantic and syntactic components of a user interface (Shneiderman \& Plaisant, 2005). In this theory, the semantic component involves the effort expended by a user to complete a given task. The syntactic component involves the effort required to understand and work with the interface, which includes interpreting the interface's feedback, and formulating and inputting commands to the interface. We propose to conceptually separate mental workload into multiple components, where the total workload required to perform a task using a computer is composed of a portion attributable to the difficulty of the task itself plus a portion attributable to the complexity of operating the user interface.

We designed a protocol to aid usability experts to measure workload as a dependent variable while a user works with an interface and/or task. The general protocol is as follows: Given an interface to evaluate and an underlying task, researchers conduct a task analysis on both the interface and task. For each subtask, they determine the cognitive subsystems that one would use while conducting the subtasks (i.e., spatial working memory, visual search, etc.). Next they gather benchmark exercises from cognitive psychology designed to elicit high and low levels of workload on the target cognitive resources associated with the interface. Researchers then run an experiment where users complete the benchmark cognition exercises, providing a measure of their brain activity while they experience high and low workload levels in their various cognitive subsystems. Users also work with the user interface that the researchers are attempting to evaluate. Lastly, researchers use fNIRS data analysis tools to find similarities and differences in the users' brain activity while working with the interface under evaluation and with the cognitive psychology exercises. 
We used this protocol while conducting an experiment to shed light on the syntactic (interface) components of workload of two specially constructed interfaces that involve users traversing through a group of web pages that are linked together while conducting an information retrieval task (Hirshfield, et al., 2009b). We kept the retrieval task constant between the two conditions, while we varied the design of the interface in each condition (Fig. $2 c$ and $2 d$ ). In the first user interface, subjects were continually informed about their location within the space, whereas in the other condition, subjects were unaware of their current location. We refer to these interface variations as the informed location UI and the uninformed location $U I$, and we hypothesize that the uninformed location UI caused subjects to experience higher loads on their spatial working memory than the informed location UI.

(a)

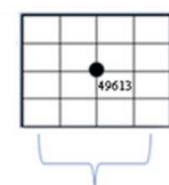

$3 \mathrm{sec}$

(b)

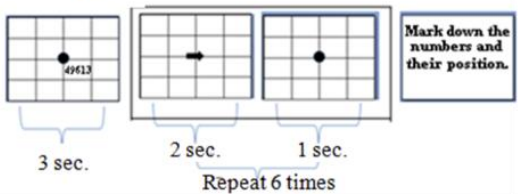

(c)
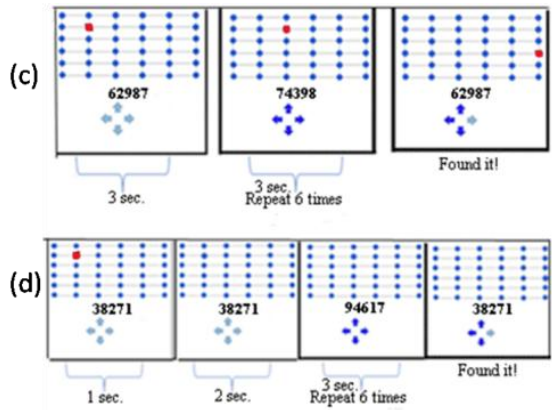

Fig.2 . Experimental Tasks. There were two benchmark tasks from cognitive psychology: (a) low spatial working memory: recall digits and location, and (b) high spatial working memory: use arrows to update location of digits. There were two interface exercises where participants had to traverse a group of linked webpages to find matching zip code, and remember current location at all times: (c) informed UI, and (d) uninformed UI.

We chose two benchmark tasks from cognitive psychology experiments that are known to involve both spatial and verbal working memory (Figure $2 \mathrm{a}$ and $2 \mathrm{~b}$ ). Both of these exercises had low verbal working memory demands (mirroring the information retrieval task). However, one of these exercises had high spatial working memory (HSWM) demands, and the other involved low spatial working memory $(L S W M)$ demands. We also had a controlled rest condition where subjects simply rested, with no workload for a set period of time. We used these tasks to provide us with benchmark brain activity for each subject where the spatial WM needed to complete the rest task was less than that needed to complete the LSWM task, which was less than that needed to complete the HSWM task.

A randomized block design with nine trials was used during the experiment. Ten subjects ( 6 female) completed the experiment. Results indicated that we could distinguish between the benchmark cognition tasks, the controlled rest, and the two user interface variations conditions using analysis of variance (ANOVA) with $95 \%$ confidence on one or both sides of subjects' heads (Hirshfield, et al., 2009b). 
We expected the uninformed UI to cause higher spatial WM load than the informed UI. We used hierarchical clustering with a Euclidian distance measure to cluster our data, which helped to validate this expectation. Clustering results showed that for $90 \%$ of subjects, the uninformed UI was grouped closer to benchmark tasks of HSWM load than the informed UI (Hirshfield, et al., 2009b). Therefore, we established that the informed UI caused a lower load on users' spatial working memory than the un-informed UI. This is not surprising, as web site designers are well aware of the benefits of keeping users oriented.

We expect this novel protocol to work in a similar manner with more realistic UIs, where the established cognitive psychology tasks may not parallel the user interfaces as closely as they did in this experiment. However, the fNIRS analysis algorithms will still show useful similarities and differences between brain activity induced by the interfaces and by the cognition exercises. While separating semantic and syntactic workload may not be possible in more complex UIs, evaluators can make informed changes to UI designs based on the level of workload measured in users' various cognitive resources while they work with a user interface.

These workload levels also may be useful input to an adaptive system if classified in real time. With this goal in mind we used a k-nearest-neighbor classifier and a distance metric computed via dynamic time warping to make comparisons between brain activity induced by the experiment conditions on a single trial basis. It was possible to distinguish between the benchmark LSWM tasks and the HSWM tasks with nearly $70 \%$ average accuracy, between each condition and the rest condition with nearly $80 \%$ average accuracy, and between the two UI conditions with $68 \%$ average accuracy. This shows promise for detecting workload changes in various cognitive resources in real-time adaptive interfaces.

In this study, we did not separate semantic and syntactic workload in the brain directly, but rather we constructed our UI and task so that the syntactic portion maps directly onto spatial working memory, and the semantic portion maps onto verbal working memory. We also developed an experimental protocol that merges low-level cognition experiments with high-level usability evaluation. Our experiment protocol and data analysis algorithms can help usability experts, or designers of adaptive systems, to acquire information about the cognitive load placed on users' various cognitive resources while working with a user interface.

\section{6 fNIRS Sensing During Interactive Game Play}

Moving away from traditional psychology experiments, where one can isolate, manipulate, and measure mental workload with great precision, we chose to apply our brain measurements techniques to a real user interface, that of an arcade game. The goal of the study was to distinguish between different levels of game difficulty using fNIRS data collected while subjects played a computer game (Girouard, et al., 2009). This study was a step forward, as previous work only evaluated the 
activeness of the user during video games using fNIRS (Matsuda \& Hiraki, 2006; Nagamitsu, Nagano, Tamashita, Takashima, \& Matsuishi, 2006). The study was designed to lead to adaptive games and other interactive interfaces that respond to the user's brain activity in real time, and we believe this work to be a stepping stone to using fNIRS in an adaptive user interface, in this case a passive braincomputer interface. Passive BCIs are interfaces that detect brain activity that occurs naturally during task performance for use as an additional input, in conjunction with standard devices such as keyboards and mice (Cutrell \& Tan, 2007). This is in contrast with active BCIs, which use brain activity as the primary input device, often requiring the user to be trained to generate specific brain states, which are interpreted as explicit input.

We asked participants to play the game of Pacman (Namco, Japan) for periods of thirty seconds, and then rest for thirty seconds. They completed ten sets of two levels of difficulty, an easy level and a hard level, during which their brain activity was measured using fNIRS. We also collected performance data, and analysis of this data showed a clear and significant distinction between the two levels. An objective measure of workload was collected using NASA-TLX (Hart \& Staveland, 1988), which confirmed two levels of mental workload.

We performed two analyses of the data to confirm the presence of differences in hemoglobin concentrations for each condition: a classic statistical analysis to establish the differences between conditions, and a more novel task classification to show the possibility of using this data in real-time adaptive systems.

The statistical analysis revealed that we can distinguish between subjects being active and passive in their mental state (playing versus resting), as well as between different levels of game complexity (difficulty level). The classification was consistent with those results, but indicated more difficulty at distinguishing the game levels (94\% to classify playing versus resting, while $61 \%$ for the easy versus hard levels). This could indicate that the mental process for this activity manifests itself at another, unmeasured location in the brain, or that the difference was simply not strong enough to cause changes in activation.

While some might argue that performance data is sufficient to classify the difficulty level of a game and can be obtained without interference, the goal of this study was to investigate the use of the brain measurements with fNIRS as a new input device. In a more complex problem, performance and fNIRS brain data might not be as related, e.g. if the user is working hard yet performing poorly at some point. In addition, distractions may also produce workload increases that would not obvious from monitoring game settings and performance, and thus may necessitate brain measurements. That is, a participant playing a simple game while answering difficult questions might also show brain activity relating to increased workload that would be incomprehensible based only on performance data (e.g. Nijholt, Tan, Allison, Milan, \& Graimann, 2008). In non-gaming situations, we might not have performance data like in the present case, as we don't always know what to measure. The use of the brain signal as an auxiliary input could provide better results in these situations. 


\section{Moving Towards an Adaptive fNIRS Interface}

Now that we have established a foundation for fNIRS measurements, the focus shifts to our second question: How do we use these measurements in the context of adaptive interfaces? How do we responsibly apply a measurement of workload in a useful way? To answer these questions we first need to find a class of problems where fNIRS may be helpful.

Given the temporal limitations of hemodynamic response in the brain, we are drawn towards problems where millisecond snapshots are not necessary, eliminating activities that are instant states or extremely time-sensitive. For example, users who are monitoring constant streams of data fit nicely into this framework.

From one perspective, the temporal limitation of fNIRS can be viewed as a healthy one - a slow hemodynamic response pushes us towards creating interfaces that are gentle and unobtrusive. This is important. If we aren't careful, braincomputer interfaces can suffer the same Midas Touch problems identified in eye tracking research (Jacob, 1993). It is a safe assumption that users do not expect an interface to change with every whim and daydream during the course of their workday. We must be judicious with our design decisions.

As a general rule for implicit interfaces, any visual modifications to the interface should be done carefully enough that the user hardly notices the change until he or she needs to (Fairclough, 2009). The eye is very sensitive to movement, and any adaptation that robs the attention of the user will be counterproductive to the primary task. A pop-up dialog box is a distracting response to a user in the middle of a task, even if we occasionally guess exactly what the user wants. Fading, overlaying information, or changing an application's screen real estate are options that, if done slowly enough, may impact the user's focus in a positive way. Visually altering the interface is just one response we can make to realtime fNIRS measurements. We can also choose to change the underlying information of the interface while keeping the general visualization stable.

While work in this direction is still in its infancy, we propose two prototypes to demonstrate how real-time, lightweight adaptive interfaces may be used with fNIRS measurements.

\subsection{The Stockbroker Scenario}

A stockbroker is writing a sensitive email to a client. At the same time, he is trying to track critical, minute-by-minute stock information on the same screen. While his email is of the upmost importance, he doesn't want to completely abandon the stock information. What if he were to miss a significant spike?

Unfortunately, the detailed visualization of stock data is mentally taxing to look at and prevents the broker from focusing on his email. As a solution, our stock- 
broker prototype gently changes the visualization of the data depending on the workload we associate with the emailing task.

If the stockbroker is not exerting a high mental workload on his email, we show his stock visualization as highly detailed, giving the stockbroker as much information as possible (Figure 3, left). If the stockbroker is working exceptionally hard at his email and cannot afford to be distracted by complex stock information, we simply lower the level of detail. The broker will still recognize major changes in the data without getting bogged down in the details (Figure 3, right).

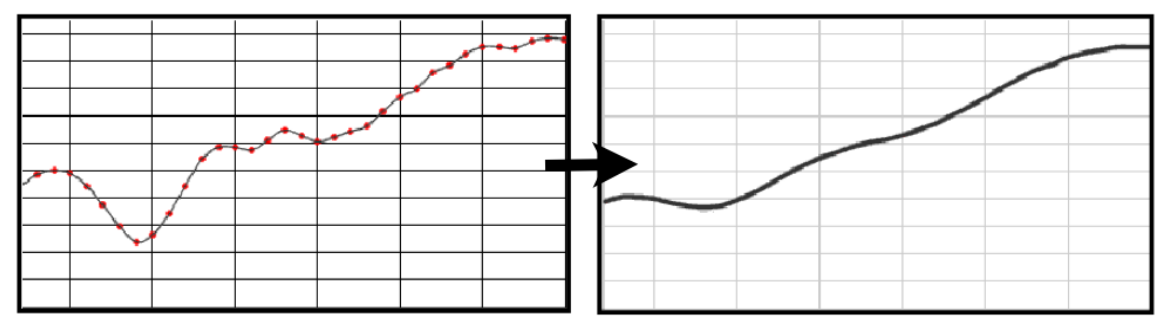

Fig 3. An example of high detailed graph (left), and one of low detail (right).

\subsection{Many Windows Scenario}

Users are often juggling four or five windows at once-writing a paragraph in one window, displaying a page of notes in another, glancing at an academic paper in a third, and tracking email in a fourth. While, like the stockbroker, the user may prefer not to get rid of any of the windows, their presence detracts from the primary task. The user may wish that the windows were only there when needed.

While fNIRS may not be able to take on the role of both mind-reader and taskdriver, fNIRS can help us guess which windows are most important. We can monitor the amount of workload required in each task, and fade the other windows accordingly. If glancing at email is relatively low-workload, then it may not be distracting. If keeping track of email is mentally expensive, then we can gradually fade the window. When the surrounding windows do not demand many mental resources, we may keep them transparent. In this way, we can think of them as being cheap to keep around.

\subsection{Looking Ahead}

These two scenarios offer a brief glimpse into brain-computer interfaces with fNIRS. They are gentle and unobtrusive. They are more concerned with long-term 
workload and trends than an immediate snapshot. Looking ahead, we can imagine numerous situations where fNIRS is a valuable input to adaptive interfaces. What if we could adjust teaching methods to best suit a child's learning style? What if we could dynamically filter streams of information (Twitter, RSS, email) to accommodate the current workload of the user?

\section{Conclusion}

We began this chapter by dealing with two major questions: (1) what kind of things can we measure using fNIRS? (2) how should we use this information as input to an adaptive user interface? Our response to the first question was three pronged: First, we explored the practical considerations for using fNIRS in HCI. We looked at the impact of head, facial, and muscle movement on fNIRS readings. We also took into account environmental factors such as ambient light and ambient noise. Next, we investigated the feasibility of using fNIRS as a measure of workload in general before measuring differences in syntactic and semantic workload in the brain, a measurement that helps us separate the interface and the underlying task. Finally, we measured a real interface, identifying a workload measurement that increased or decreased according to the complexity of the task (in this case, a game of Pacman). To address our second goal, we first outlined general characteristics for the design space of user interfaces that adapt based on fNIRS measurements. We then described two scenarios that could take advantage of such an interface design. From our experience, we believe that fNIRS brain sensing has great potential for both user interface evaluation and adaptive user interfaces, and will open new doors for human-computer interaction.

Acknowledgments The authors would like to thank the HCI research group at Tufts University; Michel Beaudoin-Lafon, Wendy Mackay, and the In|Situ| research group; and Desney Tan and Dan Morris at Microsoft Research. We thank the NSF (Grant Nos. IIS-0713506 and IIS0414389), the Canadian National Science and Engineering Research Council, the US Air Force Research Laboratory, and the US Army NSRDEC for support of this research. Any opinions, findings, and conclusions or recommendations expressed in this chapter are those of the authors and do not necessarily reflect the views of these organizations. 


\section{References}

Bunce, S. C., Izzetoglu, M., Izzetoglu, K., Onaral, B., \& Pourrezaei, K. (2006). Functional Near Infrared Spectroscopy: An Emerging Neuroimaging Modality. IEEE Engineering in Medicine and Biology Magazine, Special issue on Clinical Neuroengineering, 25(4), 54 - 62.

Chenier, F., \& Sawan, M. (2007). A New Brain Imaging Device Based on fNIRS. Paper presented at the BIOCAS 2007, 1-4.

Coyle, S., Ward, T., \& Markham, C. (2004). Physiological Noise in Near-infrared Spectroscopy: Implications for Optical Brain Computer Interfacing. Paper presented at the Proc. EMBS, 4540 - 4543.

Cutrell, E., \& Tan, D. S. (2007). BCI for passive input in HCI. Paper presented at the ACM CHI'08 Conference on Human Factors in Computing Systems Workshop on Brain-Computer Interfaces for HCI and Games.

Devaraj, A., Izzetoglu, M., Izzetoglu, K., \& Onaral, B. (2004). Motion Artifact Removal for fNIR Spectroscopy for Real World Application Areas. Proc. SPIE, 5588, 224-229.

Dietterich, T. G. (2002). Machine Learning for Sequential Data: A Review Structural, Syntactic, and Statistical Pattern Recognition (Vol. 2396, pp. 15-30): Springer-Verlag.

Ehlis, A.-C., Bähne, C. G., Jacob, C. P., Herrmann, M. J., \& Fallgatter, A. J. (2008). Reduced lateral prefrontal activation in adult patients with attention-deficit/hyperactivity disorder (ADHD) during a working memory task: A functional near-infrared spectroscopy (fNIRS) study. Journal of Psychiatric Research, 42(13), 1060-1067.

Fairclough, S. H. (2009). Fundamentals of physiological computing. Interacting with Computers, 21(1-2), 133-145.

Fuller, D., Sullivan, J., Essif, E., Personius, K., \& Fregosi, R. (1995). Measurement of the EMGforce relationship in a human upper airway muscle. Journal of Applied Physiology, 79(1), 270-278.

Gevins, A., \& Smith, M. (2003). Neurophysiological measures of cognitive workload during human-computer interaction. Theoretical Issues in Ergonomics Science, 4, 113-131.

Girouard, A., Solovey, E., Hirshfield, L., Chauncey, K., Sassaroli, A., Fantini, S., et al. (2009). Distinguishing Difficulty Levels with Non-invasive Brain Activity Measurements HumanComputer Interaction - INTERACT 2009 (pp. 440-452).

Grimes, D., Tan, D. S., Hudson, S. E., Shenoy, P., \& Rao, R. P. N. (2008). Feasibility and pragmatics of classifying working memory load with an electroencephalograph. Paper presented at the Proc. CHI'08, 835-844.

Guhe, M., Liao, W., Zhu, Z., Ji, Q., Gray, W. D., \& Schoelles, M. J. (2005). Non-intrusive measurement of workload in real-time. Paper presented at the 49th Annual Conference of the Human Factors and Ergonomics Society, 1157-1161.

Hart, S. G., \& Staveland, L. E. (1988). Development of NASA-TLX (Task Load Index): Results of empirical and theorical research. In P. Hancock, Meshkati, N. (Ed.), Human Mental Workload (pp. 139-183). Amsterdam.

Herrmann, M. J., Huter, T., Plichta, M. M., Ehlis, A.-C., Alpers, G. W., Mühlberger, A., et al. (2008). Enhancement of activity of the primary visual cortex during processing of emotional stimuli as measured with event-related functional near-infrared spectroscopy and eventrelated potentials. Human Brain Mapping, 29(1), 28-35.

Hirshfield, L. M., Chauncey, K., Gulotta, R., Girouard, A., Solovey, E. T., Jacob, R. J., et al. (2009a). Combining Electroencephalograph and Functional Near Infrared Spectroscopy to Explore Users' Mental Workload. Paper presented at the Proceedings of the 5th International Conference on Foundations of Augmented Cognition. Neuroergonomics and Operational Neuroscience: Held as Part of HCI International 2009, San Diego, CA, 239-247.

Hirshfield, L. M., Girouard, A., Solovey, E. T., Jacob, R. J. K., Sassaroli, A., Tong, Y., et al. (2007, 2007). Human-Computer Interaction and Brain Measurement Using Functional Near- 
Infrared Spectroscopy. Paper presented at the Proceedings of the ACM UIST'07 Symposium on User Interface Software and Technology.

Hirshfield, L. M., Solovey, E. T., Girouard, A., Kebinger, J., Jacob, R. J. K., Sassaroli, A., et al. (2009b). Brain measurement for usability testing and adaptive interfaces: an example of uncovering syntactic workload with functional near infrared spectroscopy. Paper presented at the Proceedings of the 27th international conference on Human factors in computing systems, Boston, MA, USA, 2185-2194.

Hoshi, Y. (2009). Near-Infrared Spectroscopy for Studying Higher Cognition Neural Correlates of Thinking (pp. 83-93).

Iqbal, S. T., Zheng, X. S., \& Bailey, B. P. (2004). Task-evoked pupillary response to mental workload in human-computer interaction. Paper presented at the $\mathrm{CHI}$ '04 extended abstracts on Human factors in computing systems, Vienna, Austria, 1477 - 1480.

Izzetoglu, K., Bunce, S., Onaral, B., Pourrezaei, K., \& Chance, B. (2004). Functional Optical Brain Imaging Using Near-Infrared During Cognitive Tasks. IJHCI, 17(2), 211-231.

Jacob, R. J. K. (1993). Eye Movement-Based Human-Computer Interaction Techniques: Toward Non-Command Interfaces. In H. R. H. a. D. Hix (Ed.), Advances in Human-Computer Interaction, Vol. 4 (pp. 151-190). Norwood, N.J.: Ablex Publishing Co.

John, M. S., Kobus, D., Morrison, J., \& Schmorrow, D. (2004). Overview of the DARPA Augmented Cognition Technical Integration Experiment. International Journal of HumanComputer Interaction, 17(2), 131-149.

Kok, A. (1997). Event-Related-Potential (ERP) Reflections of Mental Resources: A Review and Synthesis. . Biological Psychology, 45, 19-56.

Lee, J. C., \& Tan, D. S. (2006). Using a Low-Cost Electroencephalograph for Task Classification in HCI Research. Paper presented at the Proc. ACM Symposium on User Interface Software and Technology 2006, 81 - 90.

Mandryk, R., Atkins, M., \& Inkpen, K. (2006). A continuous and objective evaluation of emotional experience with interactive play environments. Paper presented at the Proceedings of the SIGCHI conference on Human factors in computing systems, Montreal, Quebec, Canada, 1027 - 1036.

Mappus, R. L., Venkatesh, G. R., Shastry, C., Israeli, A., \& Jackson, M. M. (2009). An fNIR Based BMI for Letter Construction Using Continuous Control. Paper presented at the Proc. CHI 2009 Extended Abstracts, 3571-3576.

Marshall, S., Pleydell-Pearce, C., \& Dickson, B. (2003). Integrating psychophysiological measures of cognitive workload and eye movements to detect strategy shifts. Paper presented at the IEEE. Proceedings of the 36th Annual Hawaii International Conference on System Sciences, $130 \mathrm{~b}$.

Matsuda, G., \& Hiraki, K. (2006). Sustained decrease in oxygenated hemoglobin during video games in the dorsal prefrontal cortex: A NIRS study of children. NeuroImage, 29(3), 706711.

Matthews, F., Pearlmutter, B. A., Ward, T. E., Soraghan, C., \& Markham, C. (2008). Hemodynamics for Brain-Computer Interfaces. Signal Processing Magazine, IEEE, 25(1), 87-94.

Millán, J. d. R., Renkens, F., Mouriño, J., \& Gerstner, W. (2004). Brain-actuated interaction. Artificial Intelligence, 159(1-2), 241-259.

Morioka, S., Yamada, M., \& Komori, T. (2008). Frontal Lobe Activity during the Performance of Spatial Tasks: fNIRS Study. J Phys Ther Sci, 20, 135-139.

Nagamitsu, S., Nagano, M., Tamashita, Y., Takashima, S., \& Matsuishi, T. (2006). Prefrontal cerebral blood volume patterns while playing video games-A near-infrared spectroscopy study. Brain \& Development, 28, 315-321.

Nijholt, A., Tan, D., Allison, B., Milan, J. d. R., \& Graimann, B. (2008). Brain-computer interfaces for hci and games. Paper presented at the CHI '08 extended abstracts on Human factors in computing systems, Florence, Italy, 3925-3928. 
Ramnani, N., \& Owen, A. M. (2004). Anterior prefrontal cortex: insights into function from anatomy and neuroimaging. Nat Rev Neurosci, 5(3), 184-194.

Shneiderman, B., \& Plaisant, C. (2005). Designing the User Interface: Strategies for Effective Human-Computer Interaction, Fourth Edition. Reading, Mass.: Addison-Wesley.

Sitaram, R., Zhang, H., Guan, C., Thulasidas, M., Hoshi, Y., Ishikawa, A., et al. (2007). Temporal classification of multichannel near-infrared spectroscopy signals of motor imagery for developing a brain-computer interface. NeuroImage, 34(4), 1416-1427.

Solovey, E. T., Girouard, A., Chauncey, K., Hirshfield, L. M., Sassaroli, A., Zheng, F., et al. (2009). Using fNIRS Brain Sensing in Realistic HCI Settings: Experiments and Guidelines. Paper presented at the ACM UIST'09 Symposium on User Interface Software and Technology, 157-166

Villringer, A., \& Chance, B. (1997). Non-Invasive Optical Spectroscopy and Imaging of Human Brain Function. Trends in Neuroscience, 20, 435-442. 